\title{
Correlation between reflectivity and photoluminescent properties of porous silicon films
}

\author{
Daisy Verma*, Firoz Khan, S.N. Singh, P.K. Singh \\ Electronic Materials Division, National Physical Laboratory (CSIR), New Delhi-110012, India
}

\begin{abstract}
A B S T R A C T
Porous silicon (PS) layers were formed on p-type, $\langle 100\rangle$ oriented, $1-5 \Omega \mathrm{cm}$ resistivity $\mathrm{Cz}$ silicon wafers by electrochemical etching in an $\mathrm{HF}: \mathrm{C}_{2} \mathrm{H}_{5} \mathrm{OH}(1: 2$ by volume) electrolyte at room temperature at a constant current density $20 \mathrm{~mA} / \mathrm{cm}^{2}$. The etching duration was varied to achieve PS layers of different morphologies and thicknesses. Both the photoluminescence (PL) and the total diffused reflectivity spectra of the PS layers were measured. It was found that for the PS layers grown for etching durations of less than $90 \mathrm{~s}$ the PL emission is insignificant and reflectivity is quite low. Such PS layers can be used as antireflection coatings (ARC) on solar cells. The PS layers formed for etching durations greater than 90 s show a significant PL emission in 500-800 nm range with peak lying in 630-660 nm wavelength range. When etching duration increases from $90 \mathrm{~s}$ to 8 min the PL intensity increases and the PL peak shows a blue shift. With further increase in etching duration the PL intensity decreases and PL peak shows a red shift. The reflectivity of the photoluminescent layers increases with etching duration showing a highest value for a sample grown for $8 \mathrm{~min}$. Further increase in etching duration up to $20 \mathrm{~min}$ the reflectivity decreases and then increases. Striking observation is that both the PL emission intensity and reflectivity in the wavelength range of $550-800 \mathrm{~nm}$ are maximum for the PS layer grown for the etching duration of $8 \mathrm{~min}$.
\end{abstract}

\section{Introduction}

Porous silicon (PS) has been studied for last 50 years and its potential for various applications has been highlighted in several reports [1-3]. PS material and devices made out of it are being studied by researchers globally. This material can be broadly classified as nanometric and micrometric. The former is attractive for nanotechnology missions to produce photoluminescence (PL) [4] and photonic devices [5]. The latter finds applications in biocatalyst surface [6], solar glass [7], and antireflection coating (ARC) in solar photovoltaics (SPV) using silicon (Si) wafers. The exhibited unique structural, optical and electronic properties have made PS the most promising material systems in areas as diverse as optoelectronic, single electron devices, sensors and cold cathode field emission displays. In the past decades, numerous interesting silicon nanostructures such as PS, the arrays of nanocone, nanopillars, nanorods and nanowires have been developed by traditional or newly invented methods, and many interesting optical or electrical properties were obtained, but the space remained for constructing and developing novel silicon nanostructures is still tremendous [8]. The first application of photoelectrochemically formed PS layers grown on Si wafers as an ARC was reported by Prasad et al. [9].

Although different structures have been suggested to describe the morphology of the p-type PS a correlation of its optical properties like reflectivity with its structures and photoluminescent property is not yet well established probably due to the complexity of the material. Attempts to correlate PL and reflectivity taking into account the structure of PS are very limited. One of the major problems is the deficiency of uniformity in the shape and size of the crystallites in PS.

In this work we have prepared different PS layers electrochemically by varying only one growth parameter, that is, the duration of PS formation, and have investigated its effect on reflectivity and PL of these layers.

\section{Experimental}

The starting material was $\mathrm{Cz}$ grown single crystal silicon wafers of size $50 \mathrm{~mm}$ diameter and $300 \mu \mathrm{m}$ thickness. Wafers were of p-type (B-doped) conductivity, $\langle 100\rangle$ orientation and $1-5 \Omega \mathrm{cm}$ resistivity. They were mechanically lapped on both sides and then textured in a solution of $\mathrm{NaOH}: \mathrm{IPA}: \mathrm{H}_{2} \mathrm{O}$ at $85{ }^{\circ} \mathrm{C}$ for 
$35 \mathrm{~min}$. An Al metal layer was deposited by thermal evaporation in vacuum and was sintered at $450{ }^{\circ} \mathrm{C}$ in hydrogen ambient for a good ohmic contact on the back side. Porous silicon was formed on the front surfaces of different silicon wafers for different etching durations at a constant anodization current density of $20 \mathrm{~mA} / \mathrm{cm}^{2}$.

The anodization was performed in a specially designed single compartment teflon cell using a two electrode arrangement. The electrolyte consisted of a mixture of $48 \% \mathrm{HF}$ and ethanol $\left(\mathrm{C}_{2} \mathrm{H}_{5} \mathrm{OH}\right)$ in a ratio of $1: 2$ by volume. Pt was used as a counter electrode. The as formed PS layers were characterized for PL, total diffused reflectivity $\left(R_{\lambda}\right)$ and surface morphology. The PL emission measurements were performed under an excitation wavelength of $405 \mathrm{~nm}$. The total diffused reflectivity measurements were carried out in 300-1200 nm wavelength range with a ShimadzuUV 3101PC spectrophotometer using an integrating sphere. Henceforth, the reflectivity would mean total diffused reflectivity unless mentioned otherwise. The morphology of the PS layers was examined by LEO 440 VP scanning electron microscope (SEM). Formation of the PS layers and all the measurements on them have been carried out at $25^{\circ} \mathrm{C}$. For measurements of refractive index and thickness the PS layers were formed on wafers chemically polished in a mixture of $\mathrm{HF}, \mathrm{HNO}_{3}$ and $\mathrm{CH}_{3} \mathrm{COOH}$ taken in ratio of 1:5:1 by volume. The measurements of refractive index and thickness were done with an ellipsometer a Gaertner model L117 using a He-Ne laser $(632.8 \mathrm{~nm})$ as the light source.

\section{Results and discussion}

Reflectivity of the PS layers as a function of wavelength in $300-1200 \mathrm{~nm}$ range with etching duration $\left(t_{\mathrm{e}}\right)$ as parameter is plotted in Fig. 1. It is noted that for $\lambda<450 \mathrm{~nm} R_{\lambda}$ is $<3 \%$ for all PS layers except the ones corresponding to the growth duration of 2-8 min. On the other hand, $R_{\lambda}>10 \%$, for all samples for $\lambda>1000 \mathrm{~nm}$ and increases further rapidly with wavelength till $\lambda>1200 \mathrm{~nm}$. Reflectivity is less than $10 \%$ at any value of $\lambda$ for etching duration of less than $90 \mathrm{~s}$ and decreases constantly for increase in etching duration from 20 to $90 \mathrm{~s}$. Minimum reflectivity $<5 \%$ in $400<\lambda<1000 \mathrm{~nm}$ range is observed for $t_{\mathrm{e}}=90 \mathrm{~s}$. For

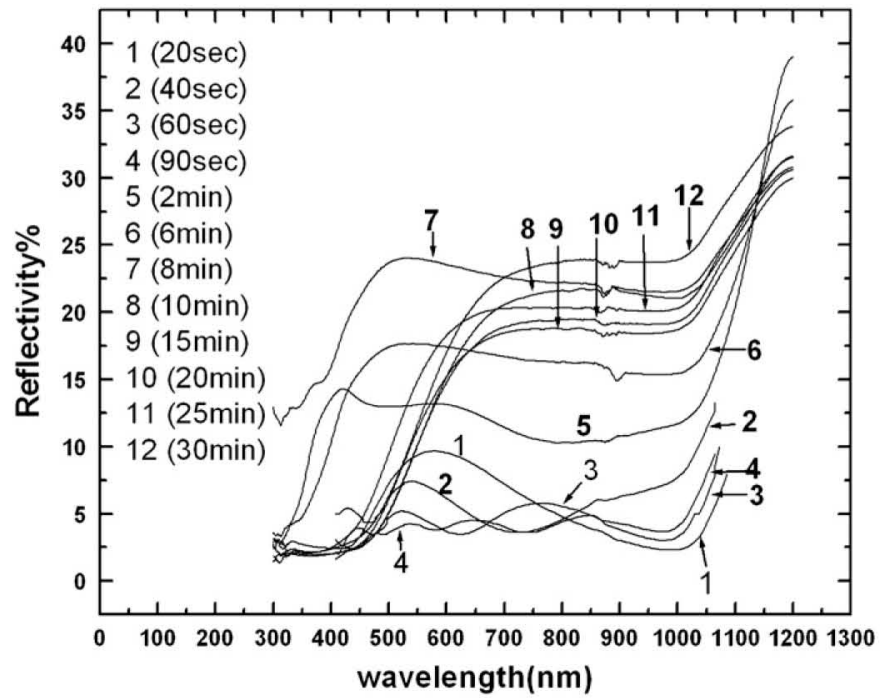

Fig. 1. Reflectance spectra for PS layers grown electrochemically for different durations varying from $20 \mathrm{~s}$ to $30 \mathrm{~min}$ at constant current density of $20 \mathrm{~mA} / \mathrm{cm}^{2}$. Curves are numbered according to etching durations. Curve 4 shows minimum reflectance and curve 7 shows the maximum reflectance in the wavelength range of 450 to $1000 \mathrm{~nm}$. $t_{\mathrm{e}}>90 \mathrm{~s}$ the reflectivity increases with $\lambda$ rather rapidly in $350<\lambda<600 \mathrm{~nm}$ range and then attains a nearly constant value till $\lambda=1000 \mathrm{~nm}$. $R_{\lambda}$ is larger for larger etching durations and its values lie between $15 \%$ and $25 \%$ for $2<t_{\mathrm{e}}<30 \mathrm{~min}$.

As shown by Singh et al. [10], the reflectivity of a $\langle 100\rangle$ oriented $\mathrm{Si}$ wafer textured in a $\mathrm{NaOH}$ solution at $\sim 80^{\circ} \mathrm{C}$ for etching durations of $10-35 \mathrm{~min}$ is nearly constant in 500$1000 \mathrm{~nm}$ wavelength range and has a value $15-25 \%$. The reflectivity values are higher than the above values for $\lambda<500$ and $\lambda>1000 \mathrm{~nm}$. Application of an ARC on the textured Si wafer reduces the reflectivity for all wavelengths below $1000 \mathrm{~nm} \mathrm{[10].}$ PS layers of suitable thicknesses have also been used as single layer ARC on Si solar cells having textured or polished front surface $[11,12]$. For ARC the refractive index $n_{\mathrm{PS}}$ of the PS layer is required to be nearly equal to $\sqrt{ } n_{\mathrm{Si}}$ where $n_{\mathrm{Si}}$ is the refractive index of bulk silicon and the optical thickness of the layer should satisfy the condition

$n_{\mathrm{PS}}^{*} d \approx(2 m+1) \lambda / 4$

where $d$ is the thickness and $n_{\mathrm{PS}} * d$ is the optical thickness of the PS layer and $m=0,1,2 \ldots$

In practice a single layer ARC is most effective if its thickness $d$ corresponds to $m=0$; the effectiveness of the ARC film decreases as $m$ increases.

For measurement of refractive index and thickness, the PS layers were created for etching durations of $20-480 \mathrm{~s}$ on $\mathrm{Cz}$ wafers chemically polished in a 1:5:1 solution of $\mathrm{HF}, \mathrm{HNO}_{3}$ and $\mathrm{CH}_{3} \mathrm{COOH}$. The measurements of refractive index $n_{\mathrm{PS}}$ and thickness $d$ of the PS layers were done at $630 \mathrm{~nm}$ using ellipsometry. Fig. 2 shows the dependence of refractive index $n_{\mathrm{PS}}$, thickness $d$ and optical thickness $n_{\mathrm{PS}}^{*} d$ of the PS layers as a function of etching duration. It can be noted that refractive index decreases from a value 2.8 at $t_{\mathrm{e}}=20 \mathrm{~s}$ to 1.74 at $t_{\mathrm{e}}=480 \mathrm{~s}$, whereas, the thickness increases from $24 \mathrm{~nm}$ at $t_{\mathrm{e}}=20 \mathrm{~s}$ to $144 \mathrm{~nm}$ at $t_{\mathrm{e}}=480 \mathrm{~s}$. The values of optical thickness $n_{\mathrm{PS}}^{*} d$ also increases with the duration of etching. The PS layers grown for $t_{\mathrm{e}}=20$ and $480 \mathrm{~s}(8 \mathrm{~min}) \mathrm{had}$ optical thicknesses values of 67 and $250 \mathrm{~nm}$, respectively. As ARC these layers should show minimum reflectivity $R_{\min }$ at 268 and $1000 \mathrm{~nm}$, respectively, corresponding to $m=0$. The latter PS layer can show $R_{\min }$ at $\sim 330 \mathrm{~nm}$ for $m=1$. For having $R_{\min }$ in $300-440 \mathrm{~nm}$ wavelength range the optical thickness of PS layer is required to be in the range $75-110 \mathrm{~nm}$ for $m=0$. Curves $1-4 \mathrm{seem}$ to satisfy these requirements well. Curves 5-7 in Fig. 1 appear to have $R_{\min }$ at $\lambda=300 \mathrm{~nm}$. These conditions cannot be satisfied for $m=0$ since the optical thickness of the PS layers for these curves

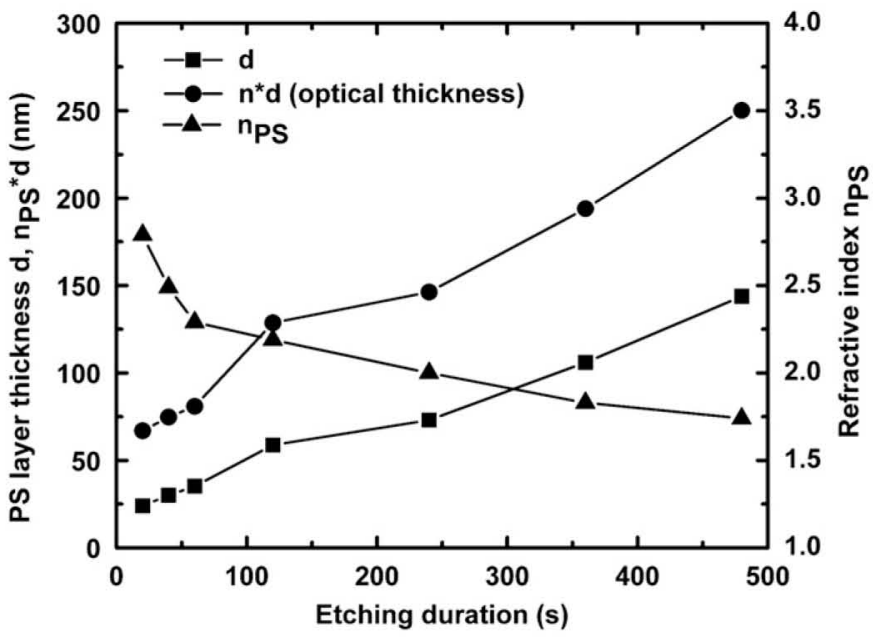

Fig. 2. Dependence of thickness, optical thickness and refractive index of PS layers on etching duration. 
are larger than $75 \mathrm{~nm}$. Therefore, it seems that the $R_{\min }$ condition is reached for $m=1$ that corresponds to an optical thickness of $\sim 225 \mathrm{~nm}$. For curves $8-12$ in Fig. $1, R_{\min }$ lie in $300-450 \mathrm{~nm}$ range and correspond to $m=1$ condition since optical thickness of these PS layers is much larger than $250 \mathrm{~nm}$.

A distinct observation that can be made from Fig. 1 is that the reflectivity increases sharply with $\lambda$ for $\lambda>1000 \mathrm{~nm}$. The earlier researchers [10] have also observed this behavior. This may be due to the very small absorption coefficient of light in silicon for $\lambda>1000 \mathrm{~nm}$ and the back reflection of such radiation from the inner back surface of the silicon wafer. This effect will be further enhanced for $\mathrm{Al}$ coated back surface as in our case.

Fig. 3 shows the photoluminescence emission spectra of porous silicon layers grown for different durations varying from $20 \mathrm{~s}$ to $30 \mathrm{~min}$. The PL was excited using a monochromatic radiation of $405 \mathrm{~nm}$ wavelength. PS layers grown for 20-90 s durations do not show any significant PL emission but the layers grown for more than 2 min show a substantial PL in $500<\lambda<800 \mathrm{~nm}$ range with peak lying between 630 and $675 \mathrm{~nm}$. The highest peak corresponds to $t_{\mathrm{e}}=8 \mathrm{~min}$ and lies at $\lambda=640 \mathrm{~nm}$.

As the etching duration is increased from 2 to $8 \mathrm{~min} \mathrm{PL}$ emission peak shows a blue shift. However, when the etching duration is increased from 8 to 30 min the PL peak shows a red shift. The explanation of such type of peak shifts of PL emission of PS samples is given by two types of models, one the most common model is quantum confinement model and second one is quantum confinement luminescent center (QCLC) model [8]. According to the quantum confinement model, PL emission is due to band to band transition and is dependent on the size of $\mathrm{Si}$ crystallites; smaller crystallites size shifts the emission peak to shorter wavelengths. According to QCLC model the radiative recombination takes place with the help of different luminescent centers of silicon oxide grown on the front surfaces of PS layers. The radiative recombination process leads to the red PL emission. The spectral behavior depends upon the shape and size distribution of Si crystallites.

Since PL spectra of most PS layers show their peaks between 630 and $650 \mathrm{~nm}$ it is important to correlate the PL emission at $\lambda=630$ and $650 \mathrm{~nm}$ with the reflectivities of different layers. Fig. 4 shows the graphs plotted between reflectivity and PL emission

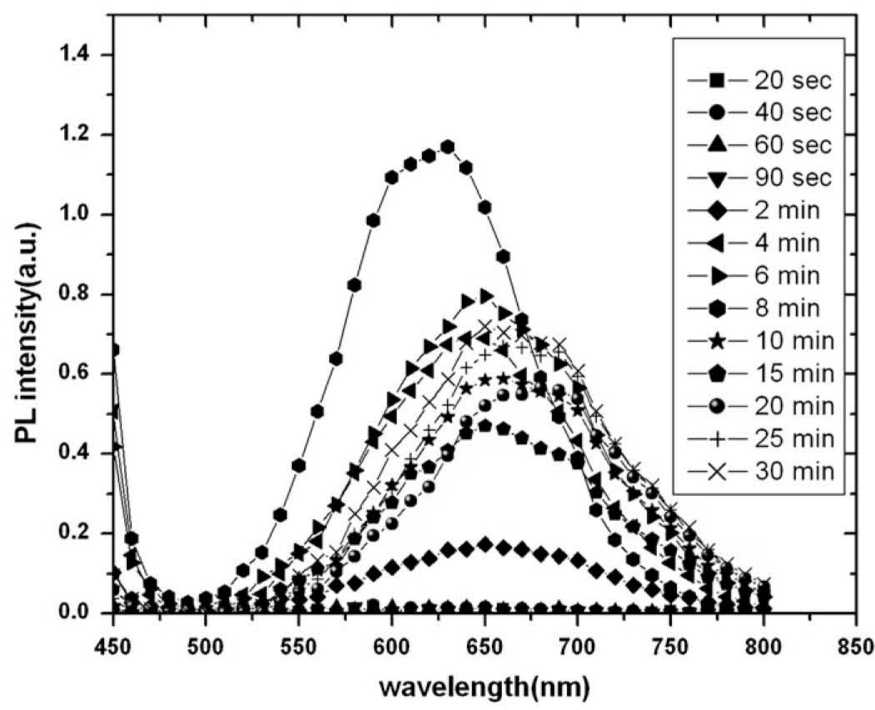

Fig. 3. Photoluminescence emission spectra of different PS layers grown electrochemically for different etching durations varying from $20 \mathrm{~s}$ to $30 \mathrm{~min}$ at constant current density of $20 \mathrm{~mA} / \mathrm{cm}^{2}$.

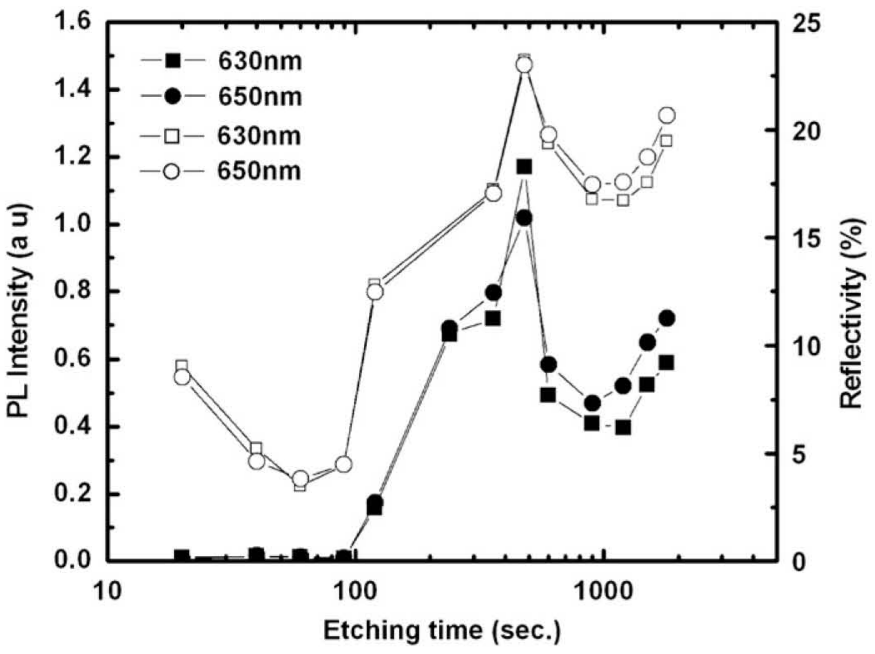

Fig. 4. Plot of photoluminescence and reflectivity with etching duration of PS layers at wavelengths 630 and $650 \mathrm{~nm}$.

intensity of all PS layers as a function of etching duration for two wavelengths $\lambda=630$ and $650 \mathrm{~nm}$. When the etching duration varies from 20 to $90 \mathrm{~s}$ there is no significant PL emission and the reflectivity is also very low. It shows that a PS layer grown for such a duration is non-photoluminescent and is suitable for application as ARC on Si solar cells. Chakravarty et al. [11] have also grown antireflection PS layers under similar conditions, which were $\sim 70 \mathrm{~nm}$ thick and had refractive index $\sim 1.9$. Fig. 4 shows that as etching duration is increased PL intensity and reflectivity both increase and both attain maximum values for the PS layer grown for $8 \mathrm{~min}$. For further increase in etching duration reflectivity decreases and so does the PL emission intensity till $t_{\mathrm{e}}=15 \mathrm{~min}$. Beyond $t_{\mathrm{e}}=15 \mathrm{~min}$ both PL and reflectivity increase with etching duration. The above observation of both high reflectivity and high PL intensity for etching duration of $8 \mathrm{~min}$ cannot be explained on the basis of the usual interference of light if the PS layer is considered as a homogeneous optical medium having a refractive index between those of air and crystalline silicon. However, Asharfi and Jagdish [13] have also found in $\mathrm{ZnO}$ layers (both zincblende and wurtzite) that PL peak energies are coincident with the reflectance peaks. Both PL and reflectance peaks occurred at $3.368 \mathrm{eV}$ that corresponded to $\lambda=368.17 \mathrm{~nm}$.

The surface morphology of PS layers was observed with scanning electron microscopy. Fig. 5 shows a top view SEM micrograph of a PS layer created for etching duration of 8 min on a textured monocrystalline p-type $\mathrm{Si}$ wafer of orientation $\langle 100\rangle$. The texture surface had straight square pyramids of height 5$10 \mu \mathrm{m}$ before formation of the PS layer. It can be seen that the vertices of the pyramids have been etched away during the electrochemical etching and it is the frustums of the pyramids that are visible in Fig. 5. It indicates that the rate of electrochemical etching may have been more at the projected portions of the silicon surface, such as, the vertices of the pyramids, facing the Pt electrode. The top surface of the pyramid frustum contains micropores that make the PS layer.

The porosity of the PS layers, measured by gravimetric method, is shown as a function of etching duration in Fig. 6. The maximum PL emission was found for the layer created for $8 \mathrm{~min}$, which had a porosity of $\sim 75 \%$. It can be noted that initially porosity increases at a fast rate but slows down after attaining a certain value, which in the present case is $\sim 65 \%$. This value was achieved for $\sim 90 \mathrm{~s}$ etching duration. All photoluminescent PS layers had porosities higher than this threshold value. 


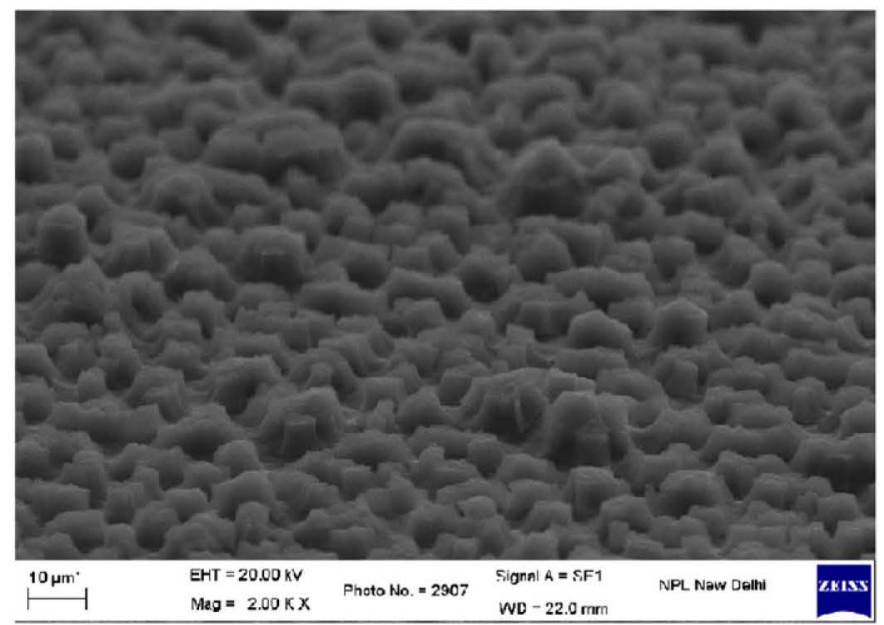

Fig. 5. SEM micrograph of PS layer formed for etching duration of $8 \mathrm{~min}$ on textured monocrystalline p-type Si wafer.

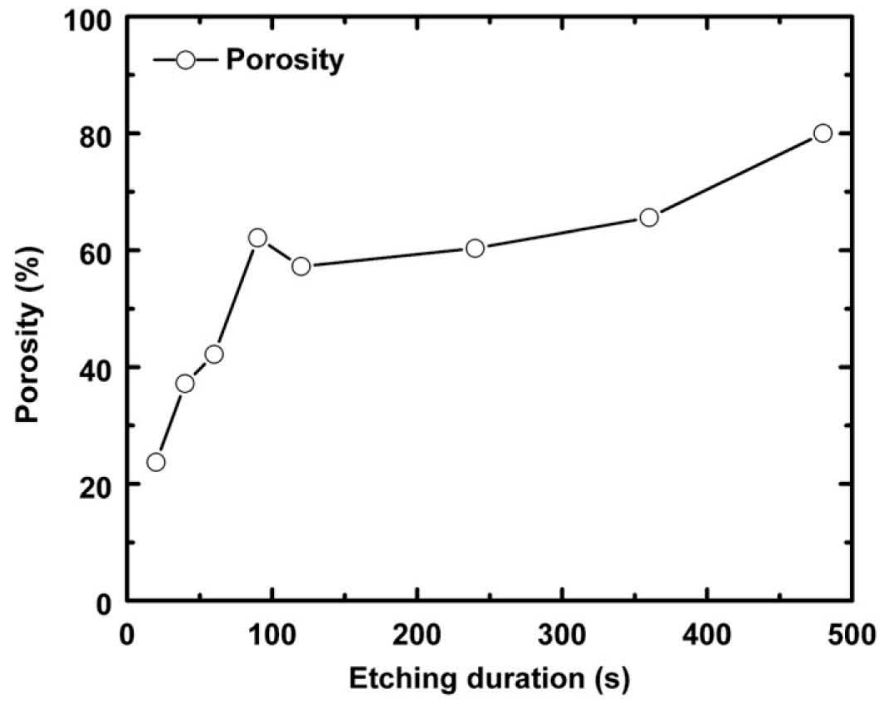

Fig. 6. Plot of porosity of PS layer vs. etching duration at constant current density of $20 \mathrm{~mA} / \mathrm{cm}^{2}$.

\section{Conclusion}

We have studied the effect of the duration of electrochemical etching on the reflectivity and PL intensity of PS layers created on $\langle 100\rangle$ oriented $\mathrm{Si}$ wafer textured in $\mathrm{NaOH}$ solution. It is found that in cases of the PS layers formed at a constant current density $20 \mathrm{~mA} / \mathrm{cm}^{2}$ for etching durations between 20 and $90 \mathrm{~s}$, the PL is insignificant and reflectivity is low. Minimum reflectivity is found for the PS layer formed for duration of $90 \mathrm{~s}$. The PS layers formed for time periods greater than $90 \mathrm{~s}$ show significant PL emission spectra with peak lying between 630 and $660 \mathrm{~nm}$ wavelengths.
When etching duration increases from $90 \mathrm{~s}$ to $8 \mathrm{~min}$ the PL intensity increases and the PL peak shows a blue shift. However, for further increase in etching duration the PL intensity decreases and PL peak shows a red shift. The reflectivity of the photoluminescent layer increases with increase in the etching duration showing a highest value for the layer grown for $8 \mathrm{~min}$. The vertices of the straight pyramids of the textured Si wafers are etched away during the electrochemical etching and frustums of the pyramids are formed. The pyramid frustums contain micropores on their surface which make the PS layer. For further increase in the etching duration the reflectivity of photoluminescent layer decreases up to 20 min duration and then increases. Striking observation is that both PL intensity and reflectivity in the wavelength range of $550-800 \mathrm{~nm}$ are maximum for PS layer formed for the durations of $8 \mathrm{~min}$.

\section{Acknowledgement}

We thank Dr. S.N. Sharma for PL measurements and Dr. M. Kar for reflectance measurements. Authors S.N. Singh, Daisy Verma and Firoz Khan also thank CSIR for the financial support for these studies.

\section{References}

[1] Y.S. Tsuo, Y. Xiao, M.J. Heben, X. Wu, F.J. Pern, S.K. Debin, Potential application of porous silicon in photovoltaics, in: Proceedings of the 23rd IEEE-PVSC, Piscalaway, NJ, 1993, pp. 287-293.

[2] S.M. Vernon, N.M. Kalkhoran, H.P. Maruska, W.D. Halverson, Proceedings of the First World Conference and Exhibition on Photovoltaics Solar Energy Conversion, Hilton Waikoloa Village Wakoloa, Hawaii, 1994, pp. 1583-1586.

[3] L. Stalmans, J. Pootrmans, H. Bender, M. Caymax, K. Said, E. Vazasonyi, J. Nijs, R. Mertens, Porous silicon in crystalline silicon solar cells: a review and the effect on the internal quantum efficiency, Prog. Photovoltaics 6 (1998) 233-246.

[4] V. Parkhulik, Porous silicon-mechanisms of growth and application, Solid State Electron. 43 (1999) 1121-1141.

[5] W.H. Zheng, P. Pece, Q.M. Sun, M. Gal, Broad band laser mirror made from porous silicon, Appl. Phys. Lett. 84 (2004) 3519-3522.

[6] M. Bengston, S. Ekstrom, J. Dratt, A. Collins, E. Csoregi, G. Marko, T. Laurell, Application of microstructured porous silicon as biocatalytic surface, Phys Status Solidi (a) 182 (2000) 495-504.

[7] C. Ballif, J. Dicker, D. Borchert, T. Hofman, Solar glass with industrial porous $\mathrm{SiO}_{2}$ antireflection coating: measurements of photovoltaic module properties improvement and modeling of yearly energy yield gain, Sol. Energy Mater. Sol. Cells 82 (2004) 331-344.

[8] H.J. Xu, X.J. Li, Silicon nanoporous pillar array: a silicon hierarchical structure with high light absorption and triple band photoluminescence, Opt. Express 16 (2008) 2933-2941.

[9] A. Prasad, S. Balakrishana, S.K. Jain, G.C. Jain, Porous silicon oxide antireflection coating for solar cells, J. Electrochem. Soc. 129 (1982) 596-599.

[10] P.K. Singh, R. Kumar, M. Lal, S.N. Singh, B.K. Das, Effectiveness of anisotopic etching of silicon in aqueous alkaline solutions, Sol. Energy Mater. Sol. Cells 70 (2001) 103-113.

[11] B.C. Chakravarty, J. Tripathy, A.K. Sharma, R. Kumar, K.N. Sood, S.B. Samanta, S.N. Singh, The growth kinetics and optical confinement studies of porous $\mathrm{Si}$ for application in terrestrial Si solar cells as antireflection coating, Sol. Energy Mater. Sol. Cells 91 (2007) 701-706.

[12] P. Panek, M. Lipinski, H. Czternastak, Porous silicon layer as an antireflection coating in solar cells, Optoelectron. Rev. 8 (2000) 57-59.

[13] A. Ashrafi, C. Jagdish, Review of zincblende $\mathrm{ZnO}$ : stability of metastable $\mathrm{ZnO}$ phases, J. Appl. Phys.-Focus. Rev. 102 (2007) 1-12 (071101). 